\title{
Di che colore è il mare? La rappresentazione degli spazi liquidi nelle mappae mundi medievali
}

\section{Maria Chiara Wang}

Storicamente, 1 (2005).

ISSN: 1825-411X. Art. no. 73. DOI: 10.12977/stor529

Corso di Laurea in Lettere Moderne

Tesi di Laurea in Geografia

Relatore: prof. Stefano Torresani

Correlatore: prof. Laura Federzoni

La tesi è articolata in cinque sezioni: 1) introduzione alla geografia e alla cartografia del Medioevo; 2) la rappresentazione degli spazi liquidi nelle mappae mundi medievali; 3) l'uso del colore nella raffigurazione del mare; 4) le isole e del loro valore simbolico; 5) schede analitiche dei documenti cartografici analizzati.

1 e 2) La geografia nel primo Medioevo (400-1000) è una materia 'fluida', non definibile poiché priva di uno status disciplinare autonomo entro la classificazione dei saperi. Nonostante ciò rientra nella sfera delle conoscenze del mondo, in relazione alla narrazione storica - come avviene già a partire da Agostino (secc. IV-V) e da Paolo Orosio (sec. V) -, alla physica (vd. Rabano Mauro, sec. IX) ed accanto alla geometria - arte liberale del quadrivium - (vd. Marziano Cappella, sec. V). II suo carattere è teoretico, atto a rispondere alle esigenze di una cultura letteraria e biblica. La geografia infatti è una fonte importante per l'esegesi delle Sacre Scritture, per la contemplazione e l'educazione. Nel Medioevo l'indagine geografica è 
inoltre caratterizzata da un ristagno nella tradizione (Macrobio e Cappella), dovuto alla priorità riservata alle autorità (Plinio, Solino e Sacre Scritture) rispetto all'esperienza e all'approccio scientifico. E' per questo che Kimble (1938) parla di 'Dark Age'.

All'interno del quadro geografico sopra delineato la mappa mundi medievale risulta essere una descrizione e narrazione - con fini teorici e pratici, educativi e mediatici - a partire da una prospettiva antropocentrica e teologica. La carta, rappresentazione semplificata di uno spazio organizzato, è un sistema semiotico costituito di simboli e di loci mnemonici, da leggere come un testo narrativo. In essa ritroviamo la storia completa della Cristianità, dalla Creazione al Giudizio Universale, il riferimento ai mostri e alle meraviglie del Iontano Oriente, la citazione di miti e leggende, la celebrazione del potere.

La produzione medievale delle mappae mundi avviene per lo più in ambito monastico; non esiste ancora la figura del cartografo e le stesse carte vengono designate con una pluralità di termini limitrofi e sinonimici quali: imago mundi, pictura, figura e descriptio. La realizzazione delle mappae è infatti subordinata al testo dei codici e dei manoscritti, entro cui sono collocate in qualità di apparato illustrativo.

Harley e Woodward (1987) propongono una tassonomia bipartita delle mappae mundi: ad una tetrapartizione per immagini fa seguito una tetrapartizione temporale. La prima classificazione si articola in: 1) mappae tripartite o T-O - schematiche e non schematiche -; 2) mappae a zone; 3) mappae quadripartite; 4) mappae di transizione. La seconda suddivisione espone in successione le seguenti fasi storiche: 1) il periodo tardo grecoromano ed il periodo patristico (ca. 400-700); 2) il Rinascimento carolingio (ca. 700-1096); 3) l'Età delle crociate (1096-1270); 4) il periodo di transizione dal Medioevo al Rinascimento (1300-1460). 
3) II colore è un prodotto storico-sociale, una categoria culturale che varia a seconda dei diversi contesti in cui è collocata. II colore ricopre funzioni differenti: da quella decorativa, a quella informativa e simbolica. Per quanto riguarda la rappresentazione degli spazi liquidi in Europa il blu diviene il colore convenzionale solo nel secolo XVII, dopo una lenta emancipazione iniziata nel secolo XI e favorita nel secolo XII da una ri-organizzazione totale della gerarchia dei colori e dall'introduzione del blu nell'ambito religioso (vd. culto mariano) da cui fino a quel momento era stato estromesso. Alla fine del Medioevo la Riforma segna la rivalutazione definitiva di questo colore, ora percepito come espressione di bellezza, nobiltà e moralità. Ma il colore, come rileva Baci? (1995) è presente anche a livello talassonimico: ogni nome riflette la posizione da cui si riguarda l'elemento designato. Così il Verde indica l'Est, il Rosso il Sud, il Nero il Nord ed il Bianco l'Ovest. E' per tale ragione che il Mar Rosso dei greci indica lo stesso spazio liquido del Golfo Verde degli arabi. Lecoq (1998) si concentra invece sulla relazione colore-significato: nel Medioevo esiste una stretta correlazione tra i venti, gli elementi, i temperamenti, gli umori, gli stati del corpo. Ulla Ehrensvärd (1987) infine valuta l'ipotesi che la scelta del colore degli spazi liquidi sia guidata da una volontà di imitazione della natura.

4) Le isole - anarchicamente distribuite - sono spazi archetipici, sedi del meraviglioso, del fantastico, dell'immaginario, dell'estraneo e dell'inusuale, e sin dall'antichità ambientazioni predilette di miti, leggende e memorie. Le isole costituiscono il limite estremo dell'orbis terrarum, la barriera tra esso e I'Oceano infinito, e in quanto tali circoscrivono il paesaggio entro una dimensione finita e perciò rassicurante. Esse possono assumere valenze sia positive, in qualità di luoghi remoti di pace, di magia, dalla temperatura mite e dal terreno fertile, sia negative quando infestate da mostri, da popolazioni barbare e da una natura selvaggia. Anche le coste nell'immaginario collettivo medievale suscitano rappresentazioni antitetiche in quanto raffigurate sia come zone di confine e di pericolo, dove si rivela la novità, 
l'alterità, la violenza e la brutalità, sia come luoghi di approdo, ritorno o fondazione e di rovesciamento carnevalesco. Le isole e le coste suscitano quindi curiosità, dubbi, interrogativi e reazioni opposte che vanno dall'edenizzazione/idealizzazione alla demonizzazione e condanna.

La tesi è infine corredata da schede analitiche dei documenti cartografici.

Sommario

\section{PREFAZIONE}

I INTRODUZIONE

I.1 Status della geografia nel Medioevo

I.2 Contenuti della cartografia e delle mappae mundi

I.3 Classificazioni delle mappae mundi

II LA GALASSIA DEGLI SPAZI LIQUIDI

II.1 II fiume Oceano

II.2 L'Oceano e il Diluvio

II.3 I fossili marini

II.4 Le creature del mare

II.5 II mare come metafora: il viaggio e la tempesta

II.6 Gli enigmi del mare

II.7 I fiumi paradisiaci

III DI CHE COLORE E' IL MARE?

III.1 I colori nel Medioevo: luminosità ed instabilità

III.2 Blu: prodotto storico - sociale

III.3 Talassonimia

III.4 Policromia - polisemia

III.5 Difficoltà documentarie, metodologiche ed epistemologiche

IV ISOLE E LIMINA

IV.1 Isole

IV.1.1 
Britannia

IV.1.2 Irlanda (lerne o Iberna o luverna)

IV.1.3 Thule

IV.1.4 Taprobana

IV.1.5 Isole Fortunate

IV.1.6 Scandza o Scandia

IV.1.7 Rifargica

IV.1.8 Utopia

IV.2 Limina

MAPPAE TRIPARTITE - SCHEMATICHE/T-O

MAPPAE TRIPARTITE - NON SCHEMATICHE

GRANDI MAPPAE TRIPARTITE - NON SCHEMATICHE

DIAGRAMMI A ZONE

MAPPAE QUADRIPARTITE

MAPPAE DI TRANSIZIONE

MAPPAE COROGRAFICHE

CARTE NAUTICHE

ILLUSTRAZIONI

REPERTORIO CARTOGRAFICO

Bibliografia

Esempi cartografici

Beatus super Apocalypsim

JEAN MANSEL La Fleur des Histoires 


\section{Link}

\section{BEATUS SUPER APOCALYPSIM MAPPA MUNDI}

[[figure caption="MAPPA MUNDI in BEATUS SUPER APOCALYPSIM. Navarra (?), 1086, manoscritto, inchiostro e colori su pergamena, $30 \times 38 \mathrm{~cm}$,. Burgo de Osma, Archivio de la Catedral, Cod. 1, fol. 34v35."]]figures/2005/wang/wang_2005_01.jpg[[/figure]] 
JEAN MANSEL La Fleur des Histoires MAPPA MUNDI

[[figure caption="MAPPA MUNDI in JEAN MANSEL La Fleur des Histoires.

Valenciennes, 1459-1463, manoscritto, penna, inchiostro e colori su

pergamena, $30 \times 22 \mathrm{~cm}$ (carta). Bruxelles, Bibliothèque Royale de Belgique, MS. 9231, fol. 281v. Mappa attribuita a Simom

Marmion"]]figures/2005/wang/wang_2005_02.jpg[[/figure]]

\section{Indietro}

